\title{
Spanning $k$-trees of Bipartite Graphs
}

\author{
Mikio Kano* \\ Ibaraki University \\ Hitachi, Ibaraki, Japan \\ kano@mx.ibaraki.ac.jp
}
Kazuhiro Suzuki ${ }^{\ddagger}$
Kochi University
Kochi, Kochi, Japan
kazuhiro@tutetuti.jp

\author{
Kenta Ozeki ${ }^{\dagger}$ \\ National Institute of Informatics \\ Chiyoda-ku, Tokyo, Japan \\ and \\ JST, ERATO, \\ Kawarabayashi Large Graph Project \\ ozeki@nii.ac.jp \\ Masao Tsugaki \\ Tokyo University of Science \\ Shinjuku-ku, Tokyo, Japan \\ tsugaki@hotmail.com
}

Submitted: Aug 2, 2013; Accepted: Jan 6, 2015; Published: Jan 20, 2015 Mathematics Subject Classifications: 05C05

\begin{abstract}
A tree is called a $k$-tree if its maximum degree is at most $k$. We prove the following theorem. Let $k \geqslant 2$ be an integer, and $G$ be a connected bipartite graph with bipartition $(A, B)$ such that $|A| \leqslant|B| \leqslant(k-1)|A|+1$. If $\sigma_{k}(G) \geqslant|B|$, then $G$ has a spanning $k$-tree, where $\sigma_{k}(G)$ denotes the minimum degree sum of $k$ independent vertices of $G$. Moreover, the condition on $\sigma_{k}(G)$ is sharp. It was shown by Win (Abh. Math. Sem. Univ. Hamburg, 43, 263-267, 1975) that if a connected graph $H$ satisfies $\sigma_{k}(H) \geqslant|H|-1$, then $H$ has a spanning $k$-tree. Thus our theorem shows that the condition becomes much weaker if the graph is bipartite.
\end{abstract}

Keywords: spanning $k$-tree, bipartite graph

\footnotetext{
*Supported by JSPS KAKENHI Grant Number 25400187.

${ }^{\dagger}$ Supported by JSPS KAKENHI Grant Number 25871053, and by Grant for Basic Science Research Projects from The Sumitomo Foundation.

${ }^{\ddagger}$ Supported by JSPS KAKENHI Grant Number 24740068.

${ }_{\text {}}$ Supported by JSPS KAKENHI Grant Number 24740074.
} 


\section{Introduction}

In this paper, we consider finite simple graphs, which have neither loops nor multiple edges. Let $G$ be a graph with vertex set $V(G)$ and edge set $E(G)$. We write $|G|$ for the order of $G$, that is, $|G|=|V(G)|$. For a vertex $v$ of $G$, let $N_{G}(v)$ denote the neighborhood of $v$ in $G$, and denote the degree of $v$ in $G$ by $\operatorname{deg}_{G}(v)$, in particular, $\operatorname{deg}_{G}(v)=\left|N_{G}(v)\right|$. A set $X$ of vertices of $G$ is called an independent set if no two vertices of $X$ are adjacent. For two vertices $x$ and $y$ of $G$, an edge joining them is denoted by $x y$ or $y x$. For an integer $k \geqslant 2$, a tree is called a $k$-tree if its maximum degree is at most $k$. Let $\alpha(G)$ denote the independence number of $G$. The number $\sigma_{k}(G)$ is defined to be the minimum degree sum of $k$ independent vertices of $G$. Namely, for an integer $k \geqslant 1$ with $\alpha(G) \geqslant k$, we define

$$
\sigma_{k}(G):=\min _{S}\left\{\sum_{x \in S} \operatorname{deg}_{G}(x): S \text { is an independent set of size } k\right\}
$$

and $\sigma_{k}(G):=\infty$ if $\alpha(G)<k$.

We begin with some known results on spanning $k$-trees related to our theorem, and other results on a spanning $k$-tree can be found in the book [1], and papers [2], [3], [5] and others. In particular, a survey article [6] contains many current results on spanning trees including spanning $k$-trees.

The next theorem gives a sufficient condition using $\sigma_{k}(G)$ for a graph to have a spanning $k$-tree.

Theorem 1 (Win [7]). Let $k \geqslant 2$ be an integer and $G$ be a connected graph. If $\sigma_{k}(G) \geqslant$ $|G|-1$, then $G$ has a spanning k-tree.

Our main result of this paper is the following theorem, which shows that the condition on $\sigma_{k}(G)$ in the above Theorem 1 can be relaxed a lot for bipartite graphs.

Theorem 2. Let $k \geqslant 2$ be an integer, and $G$ be a connected bipartite graph with bipartition $(A, B)$ such that $|A| \leqslant|B| \leqslant(k-1)|A|+1$. If

$$
\sigma_{k}(G) \geqslant|B|
$$

then $G$ has a spanning k-tree.

The above theorem with $k=2$ was obtained by Moon and Moser.

Theorem 3 (Moon and Moser [4]). Let $G$ be a connected bipartite graph with bipartition $(A, B)$ such that $|A| \leqslant|B| \leqslant|A|+1$. If $\sigma_{2}(G) \geqslant|B|$, then then $G$ has a Hamiltonian path.

Note that the condition $|B| \leqslant(k-1)|A|+1$ is necessary for the bipartite graph $G$ to have a spanning $k$-tree since if $|B|>(k-1)|A|+1$, then $G$ cannot have a spanning $k$-tree. The degree sum condition is sharp in the following sense. Let $k \geqslant 3$ and $s \geqslant 1$ be integers, and let $A_{1}, A_{2}, B_{1}$ and $B_{2}$ be disjoint sets of vertices such that $\left|A_{1}\right|=(k-2) s+1$, 
$\left|A_{2}\right|=s,\left|B_{1}\right|=s,\left|B_{2}\right|=(k-1) s+1$. Then define a bipartite graph $G$ with bipartition $\left(A_{1} \cup A_{2}, B_{1} \cup B_{2}\right)$ and edge set $E(G)=\left\{x y: x \in A_{1}, y \in B_{1}\right\} \cup\left\{x y: x \in B_{1}, y \in\right.$ $\left.A_{2}\right\} \cup\left\{x y: x \in A_{2}, y \in B_{2}\right\}$. Then $\left|A_{1} \cup A_{2}\right|=(k-1) s+1<\left|B_{1} \cup B_{2}\right|=k s+1$ and $\sigma_{k}(G)=k s=\left|B_{1} \cup B_{2}\right|-1$. Moreover, $G$ has no spanning $k$-tree. Therefore the condition on $\sigma_{k}(G)$ in Theorem 2 is sharp.

\section{Proof of Theorem 2}

We begin with some notation. Let $T$ be a tree. We denote the set of leaves of $T$ by Leaf $(T)$. For two vertices $u$ and $v$ of $T$, there exists a unique path connecting $u$ and $v$ in $T$, and it is denoted by $P_{T}(u, v)$. Let $T$ be a rooted tree with root $w$. For a vertex $v \in V(T)-\{w\}$, the vertex adjacent to $v$ and lying on the path $P_{T}(v, w)$ is called the parent of $v$ and denoted by $v^{-}$. A vertex whose parent is $v$ is called a child of $v$. In particular, there are $\operatorname{deg}_{T}(v)-1$ children of $v$, and the set of children of $v$ is denoted by Child $(v)$. We define the total excess $t e(G ; k)$ from $k$ of a graph $G$ as

$$
\operatorname{te}(G ; k):=\sum_{v \in V(G)} \max \left\{\operatorname{deg}_{G}(v)-k, 0\right\}
$$

Thus a tree $T$ has $\operatorname{te}(T ; k)=0$ if and only if $T$ is a $k$-tree. We are ready to prove Theorem 2 .

Proof of Theorem 2. By Theorem 3, we may assume that $k \geqslant 3$ though most part of the following proof holds even if $k=2$. Let $G$ be a connected bipartite graph with bipartition $(A, B)$ that satisfies the following two conditions instead of the conditions of Theorem 2 .

$$
\begin{aligned}
& \max \{|A|,|B|\} \leqslant(k-1) \min \{|A|,|B|\}+1, \quad \text { and } \\
& \sigma_{k}(G) \geqslant \max \{|A|,|B|\} .
\end{aligned}
$$

Notice that the above two conditions and the conditions of Theorem 2 are essentially equivalent, and by these new conditions, we can assume that $w \in A$ without loss of generality, which will soon be apparent, and decrease the number of cases in case analysis. Moreover, we do not use the sizes of two partite sets until the last stage of the proof.

Suppose that $G$ has no spanning $k$-tree. Choose a spanning tree $T$ of $G$ so that

(T1) te $(T ; k)$ is as small as possible,

(T2) $\mid$ Leaf $(T) \mid$ is as small as possible, subject to (T1) and,

(T3) Leaf $(T) \cap A \neq \emptyset$ and Leaf $(T) \cap B \neq \emptyset$ if possible, subject to (T2).

Since $G$ has no spanning $k$-tree, there exists a vertex $w$ such that $\operatorname{deg}_{T}(w)=l \geqslant k+1$. Let $D_{1}, D_{2}, \ldots, D_{l}$ be the components of $T-w$. For every $1 \leqslant i \leqslant l$, let $v_{i}$ denote the vertex of $D_{i}$ adjacent to $w$ in $T$. For every $1 \leqslant i \leqslant k$, let $u_{i} \in V\left(D_{i}\right)$ be a leaf of $T$, and let $U:=\left\{u_{1}, \ldots, u_{k}\right\}$.

Without loss of generality, we may assume that $w \in A$ as mentioned above. Assume that $u_{i} \in A$ for $1 \leqslant i \leqslant m$, and $u_{i} \in B$ for $m+1 \leqslant i \leqslant k$, where it might occur that 
$m=0$ or $m=k$. We regard $D_{i}$ as a rooted tree with root $u_{i}$ for $1 \leqslant i \leqslant k$, and with root $v_{i}$ for $k+1 \leqslant i \leqslant l$ (see Figure 1 ). For every $1 \leqslant t \leqslant l$, let

$$
\begin{gathered}
X_{t}:=\bigcup_{1 \leqslant j \leqslant m, j \neq t}\left(N_{G}\left(u_{j}\right) \cap V\left(D_{t}\right)\right), \\
Y_{t}:=\bigcup_{m+1 \leqslant j \leqslant k, j \neq t}\left(N_{G}\left(u_{j}\right) \cap V\left(D_{t}\right)\right), \\
Z_{t}:=\left\{\begin{array}{lll}
\left(N_{G}\left(u_{t}\right) \cap V\left(D_{t}\right)\right) \backslash X_{t} & \text { if } 1 \leqslant t \leqslant m, \\
\left(N_{G}\left(u_{t}\right) \cap V\left(D_{t}\right)\right) \backslash Y_{t} & \text { if } m+1 \leqslant t \leqslant k, \\
\emptyset & \text { if } k+1 \leqslant t \leqslant l,
\end{array}\right.
\end{gathered}
$$

and

$$
Z_{t}^{1}:=\left\{\begin{array}{c}
\left\{z \in Z_{t}: \text { a vertex of } P_{T}\left(u_{t}, z^{-}\right) \text {is adjacent to some } u_{j} \text { in } G,\right. \\
\text { where } 1 \leqslant j \leqslant m \text { and } j \neq t\} \quad \text { if } 1 \leqslant t \leqslant m, \\
\left\{z \in Z_{t}: \text { a vertex of } P_{T}\left(u_{t}, z^{-}\right) \text {is adjacent to some } u_{j} \text { in } G,\right. \\
\quad \text { where } m+1 \leqslant j \leqslant k \text { and } j \neq t\} \quad \text { if } m+1 \leqslant t \leqslant k, \\
\emptyset \quad \text { if } k+1 \leqslant t \leqslant l \quad \text { (see Figure } 1) .
\end{array}\right.
$$

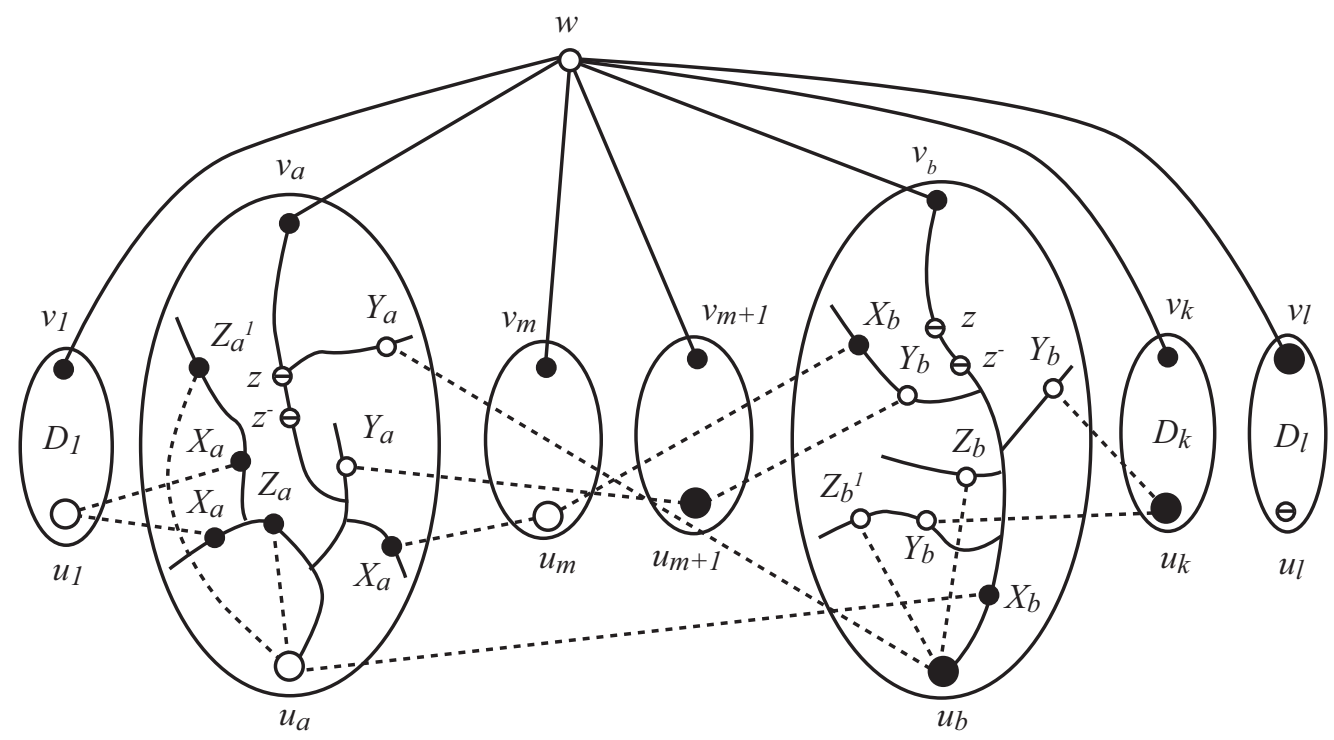

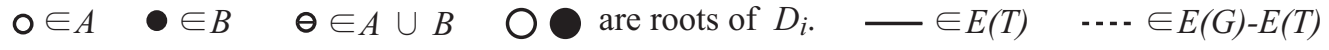

Figure 1: A spanning tree $T$ of a bipartite graph $G$ with bipartition $A \cup B$.

Then $X_{i} \subseteq B$ and $Y_{i} \subseteq A$ for all $1 \leqslant i \leqslant l$, and $Z_{i} \subseteq B$ for $1 \leqslant i \leqslant m$ and $Z_{i} \subseteq A$ for $m+1 \leqslant i \leqslant k$. We relabel indices $i$ of $D_{i}$ and rechoose $u_{i}$ so that 
(U1) $U \cap A \neq \emptyset$ and $U \cap B \neq \emptyset$ if possible and,

(U2) $X_{l} \neq \emptyset$ if possible, subject to (U1).

Let $X:=\bigcup_{1 \leqslant i \leqslant l} X_{i}, Y:=\bigcup_{1 \leqslant i \leqslant l} Y_{i}$ and $Z:=\bigcup_{1 \leqslant i \leqslant l} Z_{i}$. Then the following claim holds.

Claim 1. For every integer $1 \leqslant t \leqslant l$, let $w_{t}$ be any vertex of $D_{t}$ such that $\operatorname{deg}_{T}\left(w_{t}\right) \leqslant k-1$ or $w_{t}=v_{t}$. Then for any two distinct integers $i, j \in\{1,2, \ldots, l\}$, the following three statements hold.

(i) $w_{i} w_{j} \notin E(G)$.

(ii) If $v \in V\left(D_{i}\right)$ is adjacent to $w_{i}$ in $G-E(T)$ and if $v^{\prime} \in N_{T}(v) \cap P_{T}\left(v, w_{i}\right)$, then $v^{\prime} w_{j} \notin E(G)$.

(iii) Assume that $w_{i} \neq v_{i}, w_{j} \neq v_{j}$ and $v \in N_{G}\left(w_{i}\right) \cap V\left(D_{h}\right)$ for some $h \in\{1, \ldots, l\}-$ $\{i, j\}$. If $v^{\prime} \in N_{T}(v)$, then $v^{\prime} w_{j} \notin E(G)$.

Proof. (i). If $w_{i} w_{j} \in E(G)$ and $w_{j} \neq v_{j}$, then $T_{1}=T-w v_{i}+w_{i} w_{j}$ is a spanning tree of $G$ and $\operatorname{te}\left(T_{1} ; k\right)=\operatorname{te}(T ; k)-1$, which contradicts the choice (T1). If $w_{i}=v_{i}$ and $w_{j}=v_{j}$, then $w_{i} w_{j} \notin E(G)$ since $v_{i}$ and $v_{j}$ are both contained in $B$. Hence (i) holds.

(ii). If $v^{\prime} w_{j} \in E(G)$ and $w_{i} \neq v_{i}$, then $T_{2}=T-w v_{j}-v v^{\prime}+w_{i} v+v^{\prime} w_{j}$ is a spanning tree and $\operatorname{te}\left(T_{2} ; k\right)=\operatorname{te}(T ; k)-1$, a contradiction. If $v^{\prime} w_{j} \in E(G)$ and $w_{j} \neq v_{j}$, then $T_{2}=T-w v_{i}-v v^{\prime}+w_{i} v+v^{\prime} w_{j}$ is a spanning tree and $\operatorname{te}\left(T_{2} ; k\right)=\operatorname{te}(T ; k)-1$, a contradiction again. If $w_{i}=v_{i}$ and $w_{j}=v_{j}$, then $v^{\prime} w_{j} \notin E(G)$ since all of $v_{i}, v^{\prime}$ and $v_{j}$ are contained in $B$. Thus (ii) holds.

(iii). If $v^{\prime} w_{j} \in E(G)$, then $T_{3}=T-w v_{h}-v v^{\prime}+w_{i} v+w_{j} v^{\prime}$ is a spanning tree and $\operatorname{te}\left(T_{3} ; k\right)=\operatorname{te}(T ; k)-1$, a contradiction. Therefore Claim 1 holds.

By Claim 1 (i) and by choosing $w_{t}=u_{t}$, we have that $U$ is an independent set of $G$. Furthermore, by Claim 1 (i), we obtain the following claim.

Claim 2. For all $v \in X \cup Y$, $\operatorname{deg}_{T}(v) \geqslant k$.

Claim 3. For each $1 \leqslant i \leqslant l$, the following statements hold.

(i) $\left(\left\{v_{i}\right\} \cup \operatorname{Leaf}\left(D_{i}\right)\right) \cap\left(X_{i} \cup Y_{i}\right)=\emptyset$.

(ii) $\left(\left\{v_{i}\right\} \cup \operatorname{Leaf}\left(D_{i}\right)\right) \cap Z_{i}^{1}=\emptyset$.

Proof. (i). The statement (i) follows immediately from Claim 1 (i).

(ii). Suppose that there exists a vertex $v \in\left(\left\{v_{p}\right\} \cup\right.$ Leaf $\left.\left(D_{p}\right)\right) \cap Z_{p}^{1}$ for some $1 \leqslant p \leqslant l$. Since $Z_{i}^{1}=\emptyset$ for each $k+1 \leqslant i \leqslant l$, we have $p \in\{1, \ldots, k\}$. Assume $p \in\{1, \ldots, m\}$. Then $v \in B$, and there exist two vertices $x \in X_{p} \cap V\left(P_{T}\left(u_{p}, v^{-}\right)\right)$and $u_{q} \in U$ with $1 \leqslant q \neq p \leqslant m$ that are adjacent in $G$. Let $x^{\prime} \in \operatorname{Child}(x) \cap V\left(P_{T}\left(u_{p}, v^{-}\right)\right)$. Then $T_{1}=T-w v_{p}-x x^{\prime}+u_{p} v+x u_{q}$ is a spanning tree of $G$ and $\operatorname{te}(T ; k)>\operatorname{te}\left(T_{1} ; k\right)$ since $v \in\left\{v_{p}\right\} \cup \operatorname{Leaf}\left(D_{p}\right)$. This contradicts (T1). We can similarly derive a contradiction in the case $p \in\{m+1, \ldots, k\}$. Hence Claim 3 holds. 
By Claim 2, it follows that $\mid$ Child $(v) \mid \geqslant k-1$ for all $v \in X \cup Y$. For each $x \in X$, choose a set $C_{\text {ild }}(x)$ of $k-1$ children of $x$, and let

$$
Q(x):=\{x\} \cup \text { Child }_{k-1}(x) .
$$

Similarly, for each $y \in Y$, we define

$$
R(y):=\{y\} \cup \operatorname{Child}_{k-1}(y),
$$

where $C_{h i l d}(y)$ is a set of $k-1$ children of $y$. Then

$$
|Q(x) \cap A|=|R(y) \cap B|=k-1 \quad \text { and } \quad|Q(x) \cap B|=|R(y) \cap A|=1
$$

for every $x \in X$ and $y \in Y$. By Claim 3 (ii), Child( $z) \neq \emptyset$ for every $z \in Z_{i}^{1}$, where $1 \leqslant i \leqslant k$. For every $z \in Z_{i}$ with $1 \leqslant i \leqslant k$, let $z^{*}=z^{-}$if $z \in Z_{i}-Z_{i}^{1}$; otherwise, let $z^{*} \in \operatorname{Child}(z)$. Let

$$
S(z):=\left\{z, z^{*}\right\} \quad \text { for every } z \in Z \text {. }
$$

Claim 4. For every $z \in Z, \operatorname{deg}_{T}\left(z^{-}\right) \leqslant 2$.

Proof. Suppose that there exists a vertex $z \in Z_{i}$ such that $\operatorname{deg}_{T}\left(z^{-}\right) \geqslant 3$ for some $i$. Then $T_{1}=T-z z^{-}+z u_{i}$ is a spanning tree of $G$ and $\operatorname{te}(T ; k) \geqslant \operatorname{te}\left(T_{1} ; k\right)$ and $\mid$ Leaf $(T)|>| \operatorname{Leaf}\left(T_{1}\right) \mid$. This contradicts (T1) or (T2). Hence Claim 4 holds.

Claim 5. For all $x_{1}, x_{2} \in X, y_{1}, y_{2} \in Y$ and $z_{1}, z_{2} \in Z$ with $x_{1} \neq x_{2}, y_{1} \neq y_{2}, z_{1} \neq z_{2}$, the following holds.

(i) $Q\left(x_{1}\right) \cap Q\left(x_{2}\right)=\emptyset, R\left(y_{1}\right) \cap R\left(y_{2}\right)=\emptyset$ and $S\left(z_{1}\right) \cap S\left(z_{2}\right)=\emptyset$.

(ii) $Q\left(x_{1}\right) \cap R\left(y_{1}\right)=\emptyset, Q\left(x_{1}\right) \cap S\left(z_{1}\right)=\emptyset$ and $R\left(y_{1}\right) \cap S\left(z_{1}\right)=\emptyset$.

Proof. (i). Obviously, $Q\left(x_{1}\right) \cap Q\left(x_{2}\right)=\emptyset$ for all $x_{1} \neq x_{2} \in X$ since $x_{1}$ and $x_{2}$ are not adjacent in $T$. Similarly, $R\left(y_{1}\right) \cap R\left(y_{2}\right)=\emptyset$ for each $y_{1} \neq y_{2} \in Y$. Suppose that $S\left(z_{1}\right) \cap S\left(z_{2}\right) \neq \emptyset$ for some $z_{1} \neq z_{2} \in Z$, which implies $z_{1}^{*}=z_{2}^{*}$. By the definition of $Z_{i}$, we have $z_{1}, z_{2} \in Z_{h}$ for some $1 \leqslant h \leqslant k$. By the symmetry of $z_{1}$ and $z_{2}$, we may assume that (a) $z_{1}, z_{2} \in Z_{h}^{1}$, (b) $z_{1} \in Z_{h}^{1}$ and $z_{2} \in Z_{h}-Z_{h}^{1}$, or (c) $z_{1}, z_{2} \in Z_{h}-Z_{h}^{1}$.

First, suppose that (a) holds. Then $z_{1}^{*} \in \operatorname{Child}\left(z_{1}\right)$ and $z_{2}^{*} \in \operatorname{Child}\left(z_{2}\right)$. Hence $z_{1}^{*} \neq z_{2}^{*}$, a contradiction. Next, suppose that (b) holds. Then $z_{2}^{-}=z_{2}^{*}=z_{1}^{*} \in \operatorname{Child}\left(z_{1}\right)$, and hence $P_{T}\left(u_{h}, z_{2}^{-}\right)=P_{T}\left(u_{h}, z_{1}\right)+z_{1} z_{2}^{-}$. Since a vertex of $P_{T}\left(u_{h}, z_{1}^{-}\right)$is adjacent to some $u_{p}$ in $G$, where $p \neq h$, it follows from the definition of $Z_{h}^{1}$ that $z_{2} \in Z_{h}^{1}$. This contradicts $z_{2} \in Z_{h}-Z_{h}^{1}$. Finally, suppose that (c) holds. Then $z_{1}^{-}=z_{2}^{-}$, which implies $\operatorname{deg}_{T}\left(z_{1}^{-}\right) \geqslant 3$. This contradicts Claim 4 .

(ii). By Claim 1 (iii), $Q\left(x_{1}\right) \cap R\left(y_{1}\right)=\emptyset$ for each $x_{1} \in X$ and $y_{1} \in Y$. Suppose that $Q\left(x_{1}\right) \cap S\left(z_{1}\right) \neq \emptyset$ for some $x_{1} \in X$ and $z_{1} \in Z$. Since $Z_{i}=\emptyset$ for $k+1 \leqslant i \leqslant l$, it follows that $x_{1} \in X_{h}$ and $z_{1} \in Z_{h}$ for some $1 \leqslant h \leqslant k$. If $h \in\{1, \ldots, m\}$, then both $x_{1}$ and $z_{1}$ are contained in $B$, and so $z_{1}^{*}=z_{1}^{-} \in \operatorname{Child}\left(x_{1}\right)$. But this implies that $z_{1} \in Z_{h}^{1}$, and so $z_{1}^{*} \in \operatorname{Child}\left(z_{1}\right)$, a contradiction. 
Assume $h \in\{m+1, \ldots, k\}$. Then $x_{1} \in B$ and $z_{1} \in A$, and $x_{1}$ is adjacent to some $u_{p}$ in $G$ with $1 \leqslant p \leqslant m$. By Claim 1 (ii), $z_{1}^{-} \neq x_{1}$. Hence $x_{1}=z_{1}^{*} \in \operatorname{Child}\left(z_{1}\right)$, which implies $z_{1} \in Z_{h}^{1}$. Then there exist two vertices $y \in V\left(P_{T}\left(u_{h}, z_{1}^{-}\right)\right)$and $u_{q}$ with $m+1 \leqslant q \neq h \leqslant k$ which are adjacent in $G$. Choose $y^{\prime} \in \operatorname{Child}(y) \cap V\left(P_{T}\left(y, z_{1}\right)\right)$. Then $T_{2}=T-w v_{h}-x_{1} z_{1}-y y^{\prime}+x_{1} u_{p}+u_{h} z_{1}+u_{q} y$ is a spanning tree of $G$ and $\operatorname{te}(T ; k)>\operatorname{te}\left(T_{2} ; k\right)$. This contradicts (T1). Hence $Q\left(x_{1}\right) \cap S\left(z_{1}\right)=\emptyset$ for each $x_{1} \in X$ and $z_{1} \in Z$. Similarly, we can show that $R\left(y_{1}\right) \cap S\left(z_{1}\right)=\emptyset$ for each $y_{1} \in Y$ and $z_{1} \in Z$. Hence Claim 5 is proved.

For every $1 \leqslant i \leqslant l$, let $Q_{i}:=\bigcup_{x \in X_{i}} Q(x), R_{i}:=\bigcup_{y \in Y_{i}} R(y), S_{i}:=\bigcup_{z \in Z_{i}} S(z)$ and $O_{i}:=V\left(D_{i}\right)-\left(Q_{i} \cup R_{i} \cup S_{i}\right)$. Let

$$
Q:=\bigcup_{1 \leqslant i \leqslant l} Q_{i}, \quad R:=\bigcup_{1 \leqslant i \leqslant l} R_{i}, \quad S:=\bigcup_{1 \leqslant i \leqslant l} S_{i} \text { and } O:=\bigcup_{1 \leqslant i \leqslant l} O_{i} .
$$

Claim 6. (i) If $X_{i} \neq \emptyset$ for some $1 \leqslant i \leqslant m$ or $k+1 \leqslant i \leqslant l$, then $O_{i} \cap A \neq \emptyset$.

(ii) $O_{i} \cap B \neq \emptyset$ for each $m+1 \leqslant i \leqslant l$.

Proof. (i) Suppose that $X_{i} \neq \emptyset$ for some $1 \leqslant i \leqslant m$ or $k+1 \leqslant i \leqslant l$. Let $r_{i}$ be the root of $D_{i}$, that is, $r_{i}=u_{i}$ for $1 \leqslant i \leqslant m$, and $r_{i}=v_{i}$ for $k+1 \leqslant i \leqslant l$. Choose $x_{i} \in X_{i}$ so that $\left|P_{T}\left(r_{i}, x_{i}\right)\right|$ is as small as possible. Note that $x_{i} \neq r_{i}$ by Claim 3 (i). Recall that $x_{i} \in X_{i} \subset B$, and so $x_{i}^{-} \in A$. By Claim 1 (iii), we obtain $x_{i}^{-} \notin R_{i}$. By the minimality of $\left|P_{T}\left(r_{i}, x_{i}\right)\right|$, we have $x_{i}^{-} \notin Q_{i} \cup S_{i}$. Thus we obtain that $x_{i}^{-} \in O_{i} \cap A$, and hence $O_{i} \cap A \neq \emptyset$.

(ii) First, assume $i \in\{k+1, \ldots, l\}$. By Claim 3 (i), we have $v_{i} \notin Q_{i}$. Since $v_{i}$ is a root of $D_{i}$, we have $v_{i} \notin R_{i}$. Since $Z_{i}=\emptyset$, it follows that $v_{i} \in O_{i} \cap B$. Next, assume $i \in\{m+1, \ldots, k\}$. If $Y_{i} \neq \emptyset$, then we can also prove the statement (ii) by a similar argument as in the statement (i). Hence we may assume $Y_{i}=\emptyset$. Thus $Z_{i}^{1}=\emptyset$, and so $S(z)=\left\{z, z^{-}\right\}$for any $z \in Z_{i}$. Suppose that $z^{-}=v_{i}$ for some $z \in Z_{i}$. Then $d_{T}\left(z^{-}\right) \geqslant 3$. This contradicts Claim 4. Hence $v_{i} \notin S_{i}$. On the other hand, by Claim 3 (i), we have $v_{i} \notin Q_{i}$. Thus we obtain $v_{i} \in O_{i} \cap B$.

Claim 7. (i) $|A|=(k-1)|X|+|Y|+|Z|+|O \cap A|+1$

(ii) $|B|=|X|+(k-1)|Y|+|Z|+|O \cap B|$

Proof. By (3) and Claim 5 (i), we have $|Q \cap A|=(k-1)|X|,|R \cap A|=|Y|$ and $|S \cap A|=|Z|$. By Claim 5 (ii), $Q, R, S, O$ and $\{w\}$ are pairwise disjoint. Thus we deduce

$$
\begin{aligned}
|A| & =|Q \cap A|+|R \cap A|+|S \cap A|+|O \cap A|+|\{w\}| \\
& =(k-1)|X|+|Y|+|Z|+|O \cap A|+1 .
\end{aligned}
$$

Similarly, we can obtain the desired equality (ii).

We now prove the theorem by considering three cases.

Case 1. $|X| \geqslant|Y|+1$.

It follows that $U \cap A \neq \emptyset$ since $X \neq \emptyset$. Moreover, the following claim holds in this case. 
Claim 8. $O \cap A \neq \emptyset$.

By the assumption of Case 1 , we have $X \neq \emptyset$. If $X_{i} \neq \emptyset$ for some $1 \leqslant i \leqslant m$ or $k+1 \leqslant i \leqslant l$, then Claim 6 (i) implies $O \cap A \neq \emptyset$. Therefore we may assume that $X_{i}=\emptyset$ for all $1 \leqslant i \leqslant m$ and $k+1 \leqslant i \leqslant l$, and thus $X_{h} \neq \emptyset$ for some $m+1 \leqslant$ $h \leqslant k$. If Leaf $\left(D_{l}\right) \cap B \neq \emptyset$, then, by exchanging the role of $D_{h}$ with $u_{h}$ and $D_{l}$ with $u_{l} \in \operatorname{Leaf}\left(D_{l}\right) \cap B$, we can obtain a contradiction to (U2). Thus we have $\operatorname{Leaf}\left(D_{l}\right) \subseteq A$. It follows from Claim 3 (i) that Leaf $\left(D_{l}\right) \cap Y_{l}=\emptyset$, and since $X_{l}=\emptyset$ and $Z_{l}=\emptyset$ (by the definition of $Z_{l}$ ), we have Leaf $\left(D_{l}\right) \subseteq O \cap A$, and hence $O \cap A \neq \emptyset$.

Assume first $m \leqslant k-1$. By the assumption of Case 1 and Claims 7 (i) and 8 , we obtain

$$
\begin{aligned}
|A|= & (k-1)|X|+|Y|+|Z|+|O \cap A|+1 \\
= & m|X|+(k-m-1)|X|+|Y|+|Z|+|O \cap A|+1 \\
\geqslant & m|X|+(k-m-1)(|Y|+1)+|Y|+|Z|+2 \\
= & m|X|+(k-m)|Y|+|Z|+(k-m)+1 \\
\geqslant & \sum_{1 \leqslant i \leqslant m}\left|N_{G}\left(u_{i}\right) \cap X\right|+\sum_{m+1 \leqslant i \leqslant k}\left|N_{G}\left(u_{i}\right) \cap Y\right| \\
& +\sum_{1 \leqslant i \leqslant k}\left|N_{G}\left(u_{i}\right) \cap Z\right|+\sum_{m+1 \leqslant i \leqslant k}\left|N_{G}\left(u_{i}\right) \cap\{w\}\right|+1 \\
= & \sum_{1 \leqslant i \leqslant k} \operatorname{deg}_{G}\left(u_{i}\right)+1 \\
\geqslant & \sigma_{k}(G)+1 .
\end{aligned}
$$

Hence $\sigma_{k}(G) \leqslant|A|-1 \leqslant \max \{|A|,|B|\}-1$. By (2), this is a contradiction.

Next assume $m=k$. By (U1), we have $\operatorname{Leaf}(T) \subseteq A$. Since $|X| \geqslant|Y|+1$, it follows that $X_{p} \neq \emptyset$ for some $1 \leqslant p \leqslant l$. Let $x \in X_{p}$. Then, there exists an integer $q$ with $1 \leqslant q \leqslant k$ and $q \neq p$ such that $x \in N_{G}\left(u_{q}\right)$. Since $T_{1}=T-w v_{p}+x u_{q}$ is a spanning tree of $G$ with te $(T ; k) \geqslant \operatorname{te}\left(T_{1} ; k\right)$, it follows from (T2) that $|\operatorname{Leaf}(T)|=\left|\operatorname{Leaf}\left(T_{1}\right)\right|$, that is, $v_{p}$ is a leaf of $T_{1}$. Therefore $\operatorname{Leaf}\left(T_{1}\right) \cap A \neq \emptyset$ and $\operatorname{Leaf}\left(T_{1}\right) \cap B \neq \emptyset$. This contradicts (T3).

Case 2. $|X| \leqslant|Y|$ and $m \geqslant 1$.

By Claim 6 (ii), we have $|O \cap B| \geqslant k-m+1$. By the assumption of Case 2 and Claim 7 (ii), we obtain

$$
\begin{aligned}
|B| & =|X|+(k-1)|Y|+|Z|+|O \cap B| \\
& =|X|+(k-m)|Y|+(m-1)|Y|+|Z|+|O \cap B| \\
& \geqslant|X|+(k-m)|Y|+(m-1)|X|+|Z|+|O \cap B| \\
& \geqslant m|X|+(k-m)|Y|+|Z|+k-m+1 \\
& \geqslant \sum_{1 \leqslant i \leqslant k}\left|N_{G}\left(u_{i}\right) \cap \bigcup_{1 \leqslant j \leqslant l} V\left(D_{j}\right)\right|+\sum_{m+1 \leqslant i \leqslant k}\left|N_{G}\left(u_{i}\right) \cap\{w\}\right|+1 \\
& \geqslant \sigma_{k}(G)+1 .
\end{aligned}
$$


Hence $\sigma_{k}(G) \leqslant|B|-1 \leqslant \max \{|A|,|B|\}-1$, a contradiction.

Case 3. $m=0$.

By (U1), Leaf $\left(D_{i}\right) \subseteq B$ for all $1 \leqslant i \leqslant l$. Note that $X=\emptyset$ since $m=0$, and hence we can ignore condition (U2) and use a symmetry among $X_{i}$ 's. Since $\operatorname{deg}_{T}(w) \geqslant k+1$, if $\operatorname{deg}_{T}(a) \geqslant k$ for all $a \in A-\{w\}$ then $|B| \geqslant(k-1)|A|+2$, a contradiction. Hence we may assume that there exists a vertex $a_{h} \in V\left(D_{h}\right) \cap A$ such that $\operatorname{deg}_{T}\left(a_{h}\right) \leqslant k-1$ for some $1 \leqslant h \leqslant l$. Without loss of generality, we may assume that $h=1$. By Claim 1 (i), $\left\{a_{1}, u_{2}, \ldots, u_{k}\right\}$ is an independent set of $G$.

We regard $D_{1}$ as a rooted tree with root $a_{1}$, and change the definitions of $Y_{i}(1 \leqslant i \leqslant l)$ and $O_{i}(2 \leqslant i \leqslant l)$ as follows;

$$
Y_{i}:=\bigcup_{2 \leqslant j \leqslant k, j \neq i}\left(N_{G}\left(u_{j}\right) \cap V\left(D_{i}\right)\right), \quad O_{i}:=V\left(D_{i}\right)-\left(N_{G}\left(a_{1}\right) \cup R_{i} \cup S_{i}\right) .
$$

Following the above change of $Y_{i}$, we also change the definition of $R_{i}(2 \leqslant i \leqslant l)$.

We first consider $D_{1}$. By Claim 1 (ii), $\left(N_{G}\left(a_{1}\right) \cap V\left(D_{1}\right)\right) \cap\left(R_{1} \cap B\right)=\emptyset$. Therefore

$$
\begin{aligned}
& \left|N_{G}\left(a_{1}\right) \cap V\left(D_{1}\right)\right|+\sum_{2 \leqslant i \leqslant k}\left|N_{G}\left(u_{i}\right) \cap V\left(D_{1}\right)\right| \\
\leqslant & \left|N_{G}\left(a_{1}\right) \cap V\left(D_{1}\right)\right|+(k-1)\left|Y_{1}\right| \\
= & \left|N_{G}\left(a_{1}\right) \cap V\left(D_{1}\right)\right|+\left|R_{1} \cap B\right| \\
\leqslant & \left|V\left(D_{1}\right) \cap B\right| .
\end{aligned}
$$

We next consider $D_{2}, \ldots, D_{l}$. By Claim 1 (iii), we have $\left(N_{G}\left(a_{1}\right) \cap V\left(D_{i}\right)\right) \cap\left(R_{i} \cap B\right)=\emptyset$. In the same proof as Claim 5 (ii), we can prove that $\left(N_{G}\left(a_{1}\right) \cap V\left(D_{i}\right)\right) \cap\left(S_{i} \cap B\right)=\emptyset$ for each $2 \leqslant i \leqslant l$.

Using the same argument in the proof of Claim 6 (ii), we can prove that $O_{i} \cap B \neq \emptyset$ for each $2 \leqslant i \leqslant l$, and hence,

$$
\begin{aligned}
& \left|N_{G}\left(a_{1}\right) \cap V\left(D_{i}\right)\right|+\sum_{2 \leqslant j \leqslant k}\left|N_{G}\left(u_{j}\right) \cap V\left(D_{i}\right)\right| \\
\leqslant & \left|N_{G}\left(a_{1}\right) \cap V\left(D_{i}\right)\right|+(k-1)\left|Y_{i}\right|+\left|Z_{i}\right| \\
= & \left|N_{G}\left(a_{1}\right) \cap V\left(D_{i}\right)\right|+\left|R_{i} \cap B\right|+\left|S_{i} \cap B\right| \\
= & \left|V\left(D_{i}\right) \cap B\right|-\left|O_{i} \cap B\right| \\
\leqslant & \left|V\left(D_{i}\right) \cap B\right|-1 .
\end{aligned}
$$

By summing above two inequalities, we deduce

$$
\begin{aligned}
\sigma_{k}(G) \leqslant & \sum_{1 \leqslant i \leqslant l}\left(\left|N_{G}\left(a_{1}\right) \cap V\left(D_{i}\right)\right|+\sum_{2 \leqslant j \leqslant k}\left|N_{G}\left(u_{j}\right) \cap V\left(D_{i}\right)\right|\right) \\
& +\sum_{2 \leqslant j \leqslant k}\left|N_{G}\left(u_{j}\right) \cap\{w\}\right| \\
\leqslant & \sum_{1 \leqslant i \leqslant l}\left|V\left(D_{i}\right) \cap B\right|-(l-1)+(k-1) \\
\leqslant & |B|-1,
\end{aligned}
$$


a contradiction. Consequently the proof is complete.

\section{References}

[1] J. Akiyama and M. Kano, Factors and factorizations of graphs, LNM 2031, Chapter 8, Springer, 2011.

[2] A. Czygrinow, G. Fan, G. Hurlbert, H.A. Kierstead and W.T.Trotter, Spanning Trees of Bounded Degree, Electron. J. Combin., 8(1):\#R33, 2001.

[3] H. Matsuda and H. Matsumura, On a $k$-tree containing specified leaves in a graph, Graphs Combin. 22(3):371-381, 2006.

[4] J. Moon and L. Moser, On Hamiltonian bipartite graphs, Israel J. Math. 1:163-165, 1963.

[5] V. Neumann-Lara and E. Rivera-Campo, Spanning trees with bounded degrees, Combinatorica 11(1):55-61, 1991.

[6] K. Ozeki and T. Yamashita, Spanning trees - A survey, Graphs Combin. 27(1):1-26, 2011.

[7] S. Win, Existenz von Gerusten mit vorgeschriebenem Maximalgrad in Graphen, Abh. Math. Sem. Univ. Hamburg, 43:263-267, 1975. 